IMECE 2005-79941

\title{
FLUID FLOW AND HEAT TRANSFER IN POWER-LAW FLUIDS ACROSS CIRCULAR CYLINDERS - ANALYTICAL STUDY
}

\author{
Waqar A. Khan, Richard J. Culham, Milan M. Yovanovich \\ Microelectronics Heat Transfer Laboratory \\ Department of Mechanical Engineering \\ University of Waterloo, Waterloo, Ontario, Canada N2L 3G1 \\ Email:wkhan@mhtlab.uwaterloo.ca
}

\begin{abstract}
An integral approach of the boundary layer analysis is employed for the modeling of fluid flow around and heat transfer from infinite circular cylinders in power-law fluids. The Von Karman-Pohlhausen method is used to solve the momentum integral equation whereas the energy integral equation is solved for both isothermal and isoflux boundary conditions. A fourth-order velocity profile in the hydrodynamic boundary layer and a thirdorder temperature profile in the thermal boundary layer are used to solve both integral equations. Closed form expressions are obtained for the drag and heat transfer coefficients that can be used for a wide range of the power-law index, and generalized Reynolds and Prandtl numbers. It is found that pseudoplastic fluids offer less skin friction and higher heat transfer coefficients than dilatant fluids. As a result, the drag coefficients decrease and the heat transfer increases with the decrease in power-law index. Comparison of the analytical models with available experimental/numerical data proves the applicability of the integral approach for power-law fluids.
\end{abstract}

\section{NOMENCLATURE}

$C_{D} \quad$ total drag coefficient

$C_{D f} \quad$ friction drag coefficient

$C_{D p} \quad$ pressure drag coefficient

$C_{f} \quad$ skin friction coefficient $\equiv 2 \tau_{w} / \rho U_{a p p}^{2}$

$C_{p} \quad$ pressure coefficient $\equiv 2 \Delta P / \rho U_{a p p}^{2}$ $c_{p} \quad$ specific heat of the fluid $[\mathrm{J} / \mathrm{kg} \cdot \mathrm{K}]$

$D \quad$ cylinder diameter $[\mathrm{m}]$

$k \quad$ thermal conductivity $[\mathrm{W} / \mathrm{m} \cdot \mathrm{K}]$

$h \quad$ average heat transfer coefficient $\left[\mathrm{W} / \mathrm{m}^{2} \cdot K\right]$

$m \quad$ consistency index for non-Newtonian viscosity $\left[\mathrm{Pa} \cdot \mathrm{s}^{n}\right]$

$n \quad$ power-law index

$N u_{D} \quad$ average Nusselt number based on the diameter of the cylinder $\equiv h D / k_{f}$

$P r_{p} \quad$ Prandtl number for power-law fluids $\equiv\left(U_{a p p} D / \alpha\right) R e_{D p}^{-2 /(n+1)}$

$P \quad$ pressure $\left[N / m^{2}\right]$

$q \quad$ heat flux $\left[\mathrm{W} / \mathrm{m}^{2}\right]$

$R e_{D p} \quad$ generalized Reynolds number based on the diameter of the cylinder $\equiv D^{n} \rho U_{\text {app }}^{2-n} / m$

$s \quad$ distance along the curved surface of the circular cylinder measured from the forward stagnation point $[m]$

$T \quad$ temperature $\left[{ }^{\circ} \mathrm{C}\right]$

$U_{a p p} \quad$ approach velocity $[\mathrm{m} / \mathrm{s}]$

$U(s) \quad$ potential flow velocity just outside the boundary layer $\equiv 2 U_{\text {app }} \sin \theta[\mathrm{m} / \mathrm{s}]$

$u \quad s$ - component of velocity in the boundary layer $[\mathrm{m} / \mathrm{s}]$

$v \quad \eta$ - component of velocity in the boundary layer $[\mathrm{m} / \mathrm{s}]$ 


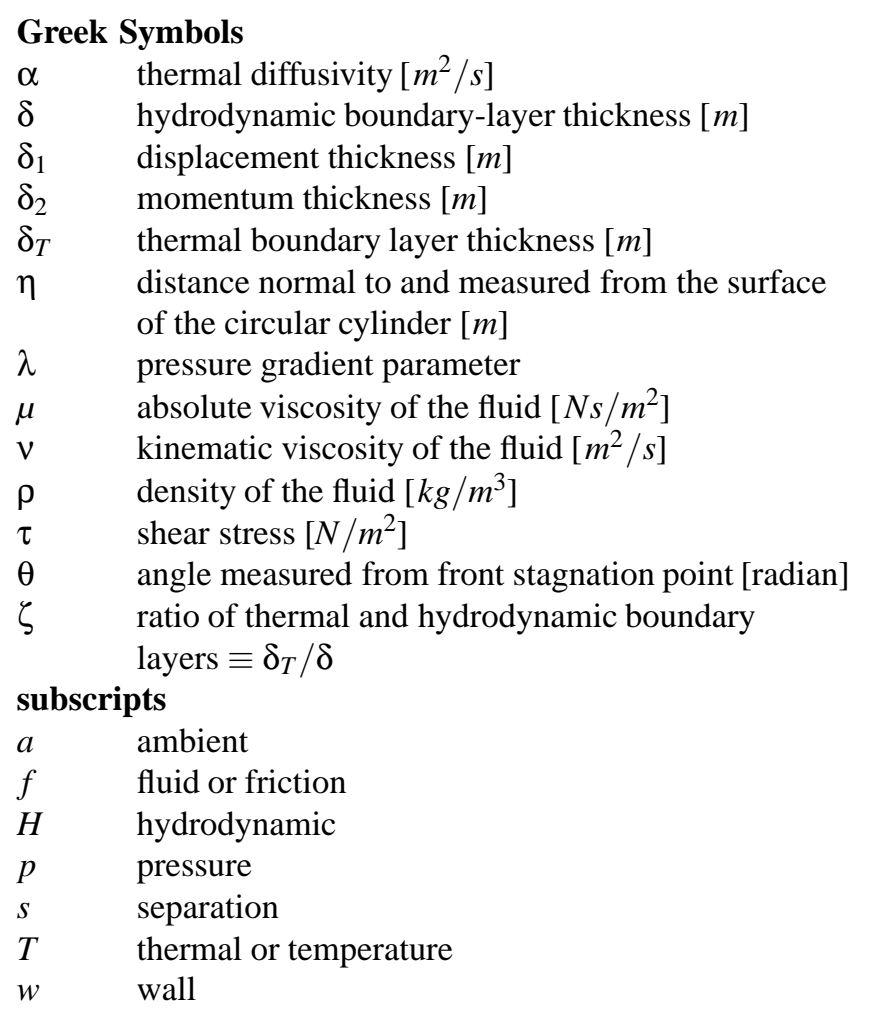

\section{INTRODUCTION}

Many practical situations need a knowledge of fluid flow around and heat transfer from horizontal cylinders subjected to crossflow of non-Newtonian fluids. These fluids are classified by different authors in different ways. One important classification is the purely viscous fluids (Cho [1]). These fluids are further subdivided in to shear thinning (or pseudoplastic, e. g. paints, glues, blood, and suspensions) and shear thickening (or dilatant, e. g. wet sand, sugar and borax solutions) fluids. In this study, fluid flow and heat transfer characteristics of these fluids across a circular cylinder are investigated using a power-law model. This model is based on the fact that both fluids exhibit a region of linear relationship between stress and strain rate when viewed on a log-log plot (Chhabra and Richardson [2]). This is why, pure viscous fluid are also called power-law fluids.

\section{Literature Review}

Fluid flow around and heat transfer from circular cylinders in crossflow to Newtonian fluids has been extensively studied theoretically, experimentally and numerically by many researchers (Khan et al. [3]) but for non-Newtonian and especially for power-law fluids these types of studies are very limited. From a theoretical point of view, Acrivos et al. [4] were the first who investigated the forced convective heat transfer from an isothermal flat plate to power-law fluids using analytical-numerical ap- proach. Later, Schowalter [5], Shah et al. [6], Acrivos et al. [7], and Lee and Ames [8] extended that work to solve 2-D boundary layer equations by using similarity transformations. Bizzell and Slattery [9] used the Von Karman-Pohlhausen integral method to analyze boundary layer on a sphere and calculated points of separation for different values of power-law index. However, no attempt was made to investigate heat transfer to power-law fluids. Wolf and Szewczyk [10] used Blasius series approach to investigate heat transfer from arbitrary cylinders to power-law fluids while Serth and Kiser [11] used Goertler series method to solve 2-D boundary layer equations for power-law fluids. Lin and Chern [12], and Kim et al. [13] presented laminar momentum boundary-layer analyses for non-Newtonian fluids by using the Merk-Chao expansion method. They obtained numerical and closed form solutions to the universal functions and then applied them to analyze the wedge flow and the flow over a circular cylinder and a sphere.

Mizushina and Usui [14] developed a theoretical framework for the laminar boundary heat transfer from a horizontal cylinder submerged in power-law fluids and expressed Nusselt number at the front stagnation point in the form

$$
N u_{D}=1.04 n^{-0.4} \operatorname{Re}_{D p}^{1 /(n+1)} \operatorname{Pr}_{p}^{1 / 3}
$$

Nakayama et al. [15], Shenoy and Nakayama [16], Nakayama [17], and Anderson [18] extended the Von Karman-Pohlhausen integral method to obtain solutions to both momentum and energy equations for power-law fluid flows past wedges, cones and axisymmetric bodies.

From experimental point of view, Shah et al. [6], Luikov et al. [19-21], James and Acosta [22], Takahashi et al. [23], Mizushina et al. [24], Mizushina and Usui [14], Kumar et al. [25], Ghosh et al. [26], Rao [27] measured local heat/mass transfer coefficients for the non-Newtonian fluids from a circular cylinder in crossflow and determined average heat transfer. Mizushina et al. [24] developed the following empirical correlation for the Nusselt number averaged over the circumference of the cylinder:

$$
N u_{D}=0.72 n^{-0.4} \operatorname{Re}_{D p}^{1 /(n+1)} \operatorname{Pr}_{p}^{1 / 3}
$$

whereas Ghosh et al. [26] developed the following empirical correlation for heat and mass transfer from a cylinder in crossflow to power-law fluids:

$$
N u_{D}=0.785 \operatorname{Re}_{D p}^{1 / 2} \operatorname{Pr}_{p}^{1 / 3} \quad \text { for } \quad 10<R e_{D p}<25000
$$

Coelho and Pinho [28-30] performed a series of experiments to study the flow of non-Newtonian fluids around a cylinder. They 
measured shedding frequency, the formation length $\left(l_{f}\right)$ and the pressure distribution around a cylinder and determined the shedding regimes and the drag coefficients.

D'Alessio and Pascal [31] investigated numerically the steady power-law flow around a circular cylinder at three different Reynolds numbers $\operatorname{Re}_{D p}=5,20$, and 40 using a firstorder accurate difference method for a fixed blockage ratio. They found that the critical Reynolds number, wake length, separation angle and drag coefficient depend on the power-law index. Chhabra et al. [32] extended that work by using a more accurate second-order finite difference method, more refined computational meshes, and greater blockage ratio and power-law index ranges in order to investigate the effect of blockage on drag coefficient, wake length, separation angle, and flow patterns over wide ranges of conditions. Agarwal et al. [33] investigated numerically the momentum and thermal boundary layers for powerlaw fluids over a thin needle under wide ranges of kinematic and physical conditions. They introduced a similarity variable and transformed the momentum and energy equations into ordinary differential equations. They reported extensive results on axial velocity profiles, shear stress and skin friction distribution on the surface of the needle, temperature and local Nusselt number variation, and momentum and thermal boundary layer thicknesses in the following ranges of conditions: $0.2 \leq n \leq 1.6,1 \leq \operatorname{Pr} \leq 1000$, $\operatorname{Re}<10^{6}$ and for three needle sizes.

In this study, an approximate method, based on the KarmanPohlhausen integral momentum and energy equations, is used to study the fluid flow and heat transfer in power-law fluids across a single circular cylinder.

\section{ANALYSIS}

Consider a uniform flow of a non-Newtonian (power-law) fluid past a fixed circular cylinder of diameter $\mathrm{D}$, with vanishing circulation around it, as shown in Fig. 1. The approaching velocity of the fluid is $U_{a p p}$ and the ambient temperature is assumed to be $T_{a}$. The surface temperature of the wall is $T_{w}\left(>T_{a}\right)$ in the case of the isothermal cylinder and the heat flux is $q$ for the isoflux boundary condition. The flow is assumed to be laminar, steady, and two dimensional. The fluid is assumed to be incompressible with constant thermophysical and rheological properties. The potential flow velocity just outside the boundary layer is denoted by $U(s)$. Using an order-of-magnitude analysis (Khan [34]), the reduced equations of continuity, momentum and energy in the curvilinear system of coordinates (Fig. 1) for a power-law fluid can be written as:

\section{Continuity:}

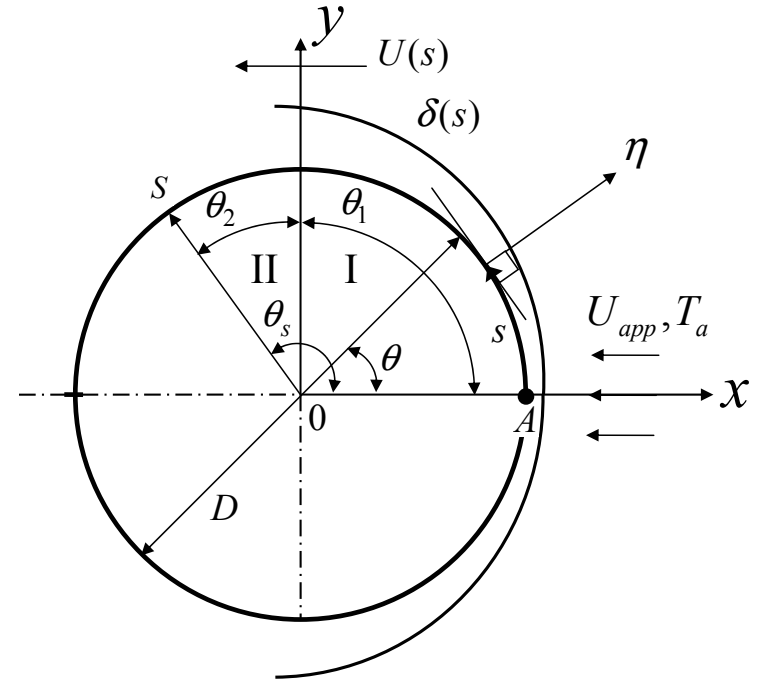

Figure 1. Flow over a circular cylinder.

s-Momentum:

$$
u \frac{\partial u}{\partial s}+v \frac{\partial u}{\partial \eta}=-\frac{1}{\rho} \frac{d P}{d s}+\frac{1}{\rho} \frac{\partial \tau_{w}}{\partial \eta}
$$

$\eta$-Momentum:

$$
\frac{d P}{d \eta}=0
$$

Energy:

$$
u \frac{\partial T}{\partial s}+v \frac{\partial T}{\partial \eta}=\alpha \frac{\partial^{2} T}{\partial \eta^{2}}
$$

with

$$
-\frac{1}{\rho} \frac{d P}{d s}=U(s) \frac{d U(s)}{d s}
$$

and

$$
\tau_{w}=\left.m\left(\frac{\partial u}{\partial \eta}\right)^{n}\right|_{\eta=0}
$$

where $m$ is a consistency index for non-Newtonian viscosity and $n$ is called power-law index, that is $<1$ for pseudoplastic, $=1$ for Newtonian, and $>1$ for dilatant fluids. 


\section{Hydrodynamic Boundary Conditions}

The assumptions of no slip boundary condition at the cylinder wall $(u=0$, at $\eta=0)$, no mass flow through the cylinder wall ( $v=0$ at $\eta=0$ ), and the potential flow just outside the boundary layer $[u=U(s)$ at $\eta=\delta(s)]$ give the following complete set of hydrodynamic conditions:

(i) at the cylinder surface, i.e., at $\eta=0$

$$
u=0 \quad \text { and } \quad \frac{\partial^{2} u}{\partial \eta^{2}}=-\frac{U(s) \frac{d U(s)}{d s}}{n \gamma\left(\frac{\partial u}{\partial \eta}\right)^{n-1}}
$$

where $\gamma=m / \rho$.

(ii) at the edge of the boundary layer, i.e., at $\eta=\delta(s)$

$$
u=U(s), \quad \frac{\partial u}{\partial \eta}=0 \quad \text { and } \quad \frac{\partial^{2} u}{\partial \eta^{2}}=0
$$

These conditions will help in determining the velocity distribution inside the boundary layer.

\section{Thermal Boundary Conditions}

The assumptions of uniform wall temperature (UWT) and uniform wall flux (UWF) boundary conditions give the following complete set thermal conditions:

(i) at the cylinder surface, i.e., at $\eta=0$

$$
\frac{\partial^{2} T}{\partial \eta^{2}}=0 \quad \text { and } \quad \begin{cases}T=T_{w} & \text { for UWT } \\ \frac{\partial T}{\partial \eta}=-\frac{q}{k_{f}} & \text { for UWF }\end{cases}
$$

(ii) at the edge of thermal boundary layer, i.e., at $\eta=\delta_{T}$

$$
T=T_{a} \quad \text { and } \quad \frac{\partial T}{\partial \eta}=0
$$

Using these thermal conditions, the temperature distributions inside the thermal boundary layer can be determined.

\section{Velocity Distribution}

Assuming a thin hydrodynamic boundary layer around the cylinder, the velocity distribution inside the boundary layer, satisfying Eqs. (10) and (11), can be approximated by a fourth order polynomial as suggested by Pohlhausen [35]:

$$
\frac{u}{U(s)}=\left(2 \eta_{H}-2 \eta_{H}^{3}+\eta_{H}^{4}\right)+\frac{\lambda}{6}\left(\eta_{H}-3 \eta_{H}^{2}+3 \eta_{H}^{3}-\eta_{H}^{4}\right)
$$

where $0 \leq \eta_{H}=\eta / \delta(s) \leq 1$ and $\lambda$ is the pressure gradient parameter, given by

$$
\lambda=\frac{\frac{d U(s)}{d s} \delta^{n+1} U(s)^{1-n}\left(2+\frac{\lambda}{6}\right)^{1-n}}{n \gamma}
$$

With the help of velocity profiles, Schlichting [36] showed that the parameter $\lambda$ is restricted to the range $-12 \leq \lambda \leq 12$.

\section{Temperature Distribution}

Assuming a thin thermal boundary layer around the cylinder, the temperature distribution in the thermal boundary layer, satisfying Eqs. (12) and (13), can be approximated by a third order polynomial

$$
\frac{T-T_{a}}{T_{w}-T_{a}}=1-\frac{3}{2} \eta_{T}+\frac{1}{2} \eta_{T}^{3}
$$

for the isothermal boundary condition and

$$
T-T_{a}=\frac{2 q \delta_{T}}{3 k_{f}}\left(1-\frac{3}{2} \eta_{T}+\frac{1}{2} \eta_{T}^{3}\right)
$$

for the isoflux boundary condition.

\section{Boundary Layer Parameters}

In dimensionless form, the momentum integral equation can be written as

$$
U^{2} \frac{d \delta_{2}}{d s}+\left(2 \delta_{2}+\delta_{1}\right) U \frac{d U}{d s}=\frac{1}{\rho} \tau_{w}
$$

where $\delta_{1}$ and $\delta_{2}$ are the displacement and momentum boundary layer thicknesses and are given by

$$
\delta_{1}=\delta \int_{0}^{1}\left[1-\frac{u}{U(s)}\right] d \eta
$$

and

$$
\delta_{2}=\delta \int_{0}^{1} \frac{u}{U(s)}\left[1-\frac{u}{U(s)}\right] d \eta
$$


Using velocity distribution from Eq. (14), Eqs. (19) and (20) can be written as:

$$
\delta_{1}=\frac{\delta}{10}\left(3-\frac{\lambda}{12}\right)
$$

and

$$
\delta_{2}=\frac{\delta}{63}\left(\frac{37}{5}-\frac{\lambda}{15}-\frac{\lambda^{2}}{144}\right)
$$

Simplifying and arranging the terms in Eq. (18), we get

$$
\frac{U \delta_{2}^{n}}{\gamma U^{n-1}} \frac{d \delta_{2}}{d s}+\left(2+\frac{\delta_{1}}{\delta_{2}}\right) \frac{\delta_{2}^{n+1} U^{1-n}}{\gamma} \frac{d U}{d s}=\left(\frac{\delta_{2}}{\delta}\right)^{n}\left(2+\frac{\lambda}{6}\right)^{n}
$$

Assuming

$$
Z=\frac{\delta_{2}{ }^{n+1} U^{1-n}}{\gamma} \quad \text { and } \quad K=Z \frac{d U}{d s}
$$

Equation (23) can be reduced to a non-linear differential equation of the first order for $Z$, which can be written as:

$$
\frac{d Z}{d s}=\frac{F}{U}
$$

where

$$
F=\frac{K\left[\frac{(n+1)\left(2+\frac{\lambda}{6}\right)}{n}-(1+3 n) \lambda\left(\frac{\delta_{2}}{\delta}\right)-(n+1) \lambda\left(\frac{\delta_{1}}{\delta}\right)\right]}{\lambda\left(\frac{\delta_{2}}{\delta}\right)}
$$

At the stagnation point $s=0, U=0$. Since $d Z / d s$ cannot be infinite, $F$ must be zero at the stagnation point. Hence

$$
\frac{(n+1)\left(2+\frac{\lambda}{6}\right)}{n}-(1+3 n) \lambda\left(\frac{\delta_{2}}{\delta}\right)-(n+1) \lambda\left(\frac{\delta_{1}}{\delta}\right)=0
$$

which gives the values of the pressure gradient parameter $\lambda$ for different values of $n$ at the stagnation point. Due to limitations of the method used in this study, no root of Eq. (26) could be found in the range $-12 \leq \lambda \leq 12$ for $n<0.895$. Bizzell and Slattery [9] could calculate the roots for $0.7358 \leq n \leq 1.0$ only whereas Mizushina and Usui [14] calculated the roots in the range $0.895 \leq n \leq 1.19$. In the present study, the values of $\lambda$ are calculated for $n \geq 0.895$. These values are plotted in Fig. 2 as a function of $n$.

Following Walz [37], the function $F$ can be approximated by a straight line

$$
F=a-b K
$$

where the constants $a$ and $b$ are determined for each power index $n$ and are correlated in to single correlations

$$
a=0.45 n^{-1.25} \quad \text { and } \quad b=6.2 n^{0.8}
$$

These correlations are valid between the stagnation point $(F=0)$ and the point of maximum velocity $(K=0)$. So Eq. (24) can be written as

$$
U(s) \frac{d Z}{d s}=a-b K
$$

Using potential flow velocity outside the boundary layer for a circular cylinder and rearranging the terms, Eq. (29) can be solved for the local dimensionless momentum thickness:

$$
\frac{\delta_{2}}{D}=\left[\frac{a(\sin \theta)^{1+b-n}}{2^{3-n} R e_{D p}} \cdot \int_{0}^{\theta}(\sin \zeta)^{b-1} d \zeta\right]^{1 /(n+1)}
$$

where $\zeta$ is a dummy variable and $R e_{D p}$ is the generalized Reynolds number for a power-law fluid and is defined as

$$
\operatorname{Re}_{D p}=\frac{D^{n} \rho U_{a p p}^{2-n}}{m}
$$

From Eq. (15), the dimensionless hydrodynamic boundary layer thickness can be written as

$$
\frac{\delta}{D}=\left[\frac{1}{R e_{D p}} \cdot \frac{n \lambda \sin ^{n} \theta}{2^{2-n} \sin 2 \theta\left(2+\frac{\lambda}{6}\right)^{1-n}}\right]^{1 /(n+1)}
$$

Solving Eq. (22) with Eq. (32) and comparing the results with the numerical values obtained from Eq. (30), one can get the values of pressure gradient parameter $\lambda$ for each position along the 


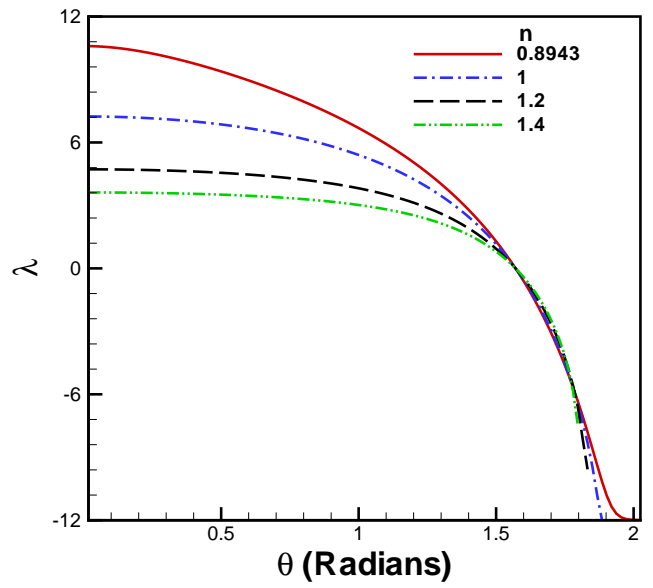

Figure 2. Behavior of Pressure Gradient Parameter for Power-Law Fluids.

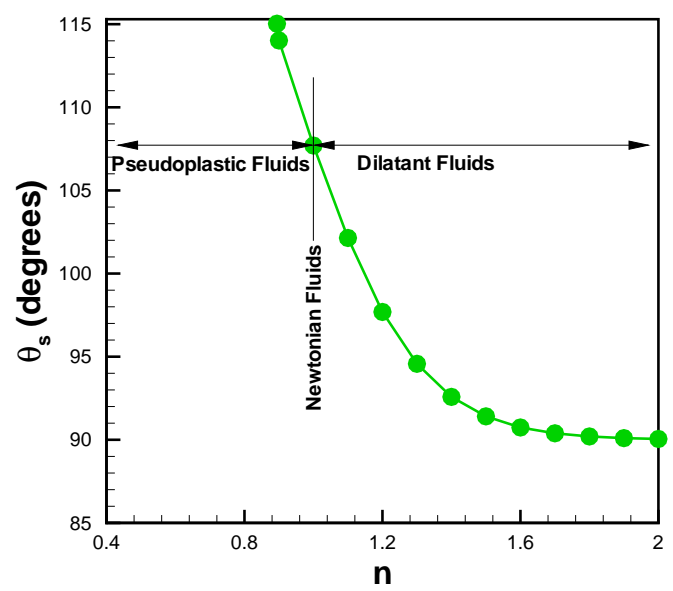

Figure 3. Angles of Separation for Power-Law Fluids.

circumference of the cylinder. These values were fitted by the least squares method for each power-law index $n$ and are shown in Fig. 2 for pseudoplastic and dilatant fluids. It shows that the value of $\lambda$ decreases with increasing $n$. Using analytical definition of the point of separation, the angle of separation was determined which depends on the nature of the fluid (pseudoplastic or dilatant) as well as on the velocity distribution inside the boundary layer. The calculated angles of separation are plotted in Fig. 3 as a function of $n$. It shows that the separation angle decreases with the increase in power-law index $n$, which is in accordance with Serth and Kiser [11].

\section{Fluid Flow}

The first parameter of interest is fluid friction which manifests itself in the form of the drag force $F_{D}$, where $F_{D}$ is the sum of the skin friction drag $D_{f}$ and pressure drag $D_{p}$. Skin friction drag is due to viscous shear forces produced at the cylinder surface, predominantly in those regions where the boundary layer is attached. In dimensionless form, it can be written as

$$
C_{f}=\frac{\tau_{w}}{\frac{1}{2} \rho U_{a p p}^{2}}
$$

Using Eqs. (9) and (14) and simplifying, we get

$$
C_{f}=\frac{2}{R e_{D p}^{1 /(n+1)}}\left[\frac{(\lambda+12) \sin \theta}{3}\right]^{n}\left[\frac{\sin 2 \theta}{2^{n-2}} \frac{\left(2+\frac{\lambda}{6}\right)^{1-n}}{n \lambda \sin ^{n} \theta}\right]^{n /(n+1)}
$$

The friction drag coefficient can be defined as

$$
\begin{aligned}
C_{D f} & =\int_{0}^{\pi} C_{f} \sin \theta d \theta \\
& =\int_{0}^{\theta_{s}} C_{f} \sin \theta d \theta+\int_{\theta_{s}}^{\pi} C_{f} \sin \theta d \theta
\end{aligned}
$$

Since the shear stress on the cylinder surface after boundary layer separation is very small, the second integral can be neglected and the friction drag coefficient can be written as

$$
C_{D f}=\int_{0}^{\theta_{s}} C_{f} \sin \theta d \theta
$$

The calculations were performed for different values of $n$ and the results are correlated in terms of $n$ and the generalized Reynolds number $R e_{D p}$ in to a single correlation

$$
C_{D f}=\frac{5.786 n^{0.32}}{\operatorname{Re}_{D p}^{1 /(n+1)}}
$$

which gives friction drag coefficient for the flow of a power-law fluid over a circular cylinder in an infinite medium. It is interesting to note here that for $n=1$, Eq. (37) gives the friction drag coefficient for the flow of Newtonian fluid over a circular cylinder (Khan, et al. [3]).

Pressure drag is due to the unbalanced pressures which exist between the relatively high pressures on the upstream surfaces and the lower pressures on the downstream surfaces. In dimensionless form, it can be written as

$$
C_{D p}=\int_{0}^{\pi} C_{p} \cos \theta d \theta
$$


where $C_{p}$ is the pressure coefficient and can be defined as

$$
C_{p}=\frac{\Delta P}{\frac{1}{2} \rho U_{a p p}^{2}}
$$

The pressure difference $\Delta P$ can be obtained by integrating $\theta$ momentum equation with respect to $\theta$. Following Shibu et al. [38], the $\theta$-momentum equation, for power-law fluids, can be written as

$$
\begin{array}{r}
u_{r} \frac{\partial u_{\theta}}{\partial r}+\frac{u_{\theta}}{r} \frac{\partial u_{\theta}}{\partial \theta}+\frac{u_{r} u_{\theta}}{r}=-\frac{1}{2 r} \frac{\partial C_{p}}{\partial \theta}+\frac{2^{n}}{R e_{D p}} \\
\cdot\left[\frac{1}{r^{2}} \frac{\partial}{\partial r}\left(r^{2} \tau_{r \theta}\right)+\frac{1}{r} \frac{\partial \tau_{\theta \theta}}{\partial \theta}\right]
\end{array}
$$

where

$$
\tau_{r \theta}=2 \eta \varepsilon_{r \theta} \quad \tau_{\theta \theta}=2 \eta \varepsilon_{\theta \theta}
$$

with

and

$$
\begin{array}{ll}
\eta=(2 \Pi)^{(n-1) / 2} & \varepsilon_{r \theta}=\frac{1}{2}\left[r \frac{\partial}{\partial r}\left(\frac{u_{\theta}}{r}\right)+\frac{1}{r} \frac{\partial u_{r}}{\partial \theta}\right] \\
\varepsilon_{\theta \theta}=\frac{1}{r} \frac{\partial u_{\theta}}{\partial \theta}+\frac{u_{r}}{r} & \Pi=\varepsilon_{r r}^{2}+\varepsilon_{\theta \theta}^{2}+2 \varepsilon_{r \theta}^{2}
\end{array}
$$

$$
u_{r}=\cos \theta\left(1-\frac{1}{r^{2}}\right) \quad u_{\theta}=-\sin \theta\left(1+\frac{1}{r^{2}}\right)
$$

In Eq. (40), Shibu et al. [38] scaled the velocity terms using $U_{a p p}$, pressure with $\frac{1}{2} \rho U_{a p p}^{2}$, radial coordinate by the radius of the cylinder $R$, the stress components by $m\left(U_{a p p} / R\right)^{n}$, and the second invariant of the rate of deformation tensor, $\Pi$ using $\left(U_{\text {app }} / R\right)^{2}$. Using derivatives of the velocity components and an order-of-magnitude analysis (Khan [34]), Eq. (40) can be reduced to

$$
\frac{\partial C_{p}}{\partial \theta}=-4 \sin 2 \theta-\frac{2^{3 n}}{R e_{D p}} \sin \theta
$$

Integrating it with respect to $\theta$, we get

$$
C_{p}=2(1-\cos 2 \theta)+\frac{2^{3 n}}{R e_{D p}}(1-\cos \theta)
$$

So, the pressure drag coefficient for the cylinder up to the separation point will be

$$
C_{D p}=\int_{0}^{\theta_{s}} C_{p} \cos \theta d \theta
$$

This pressure drag coefficient was calculated for different values of $n$ and correlated in to a single correlation in terms of $n$ and $\operatorname{Re}_{D p}$ :

$$
C_{D p}=\frac{1.26 n^{3.25}}{\operatorname{Re}_{D p}}+1.28[1-\exp (-2.4 n)]
$$

The total drag coefficient $C_{D}$ can be written as the sum of both drag coefficients

$$
C_{D}=\frac{5.786 n^{0.32}}{R e_{D p}^{1 /(n+1)}}+\frac{1.26 n^{3.25}}{R e_{D p}}+1.28[1-\exp (-2.4 n)]
$$

which agrees with the drag coefficient of a Newtonian fluid ( $n=$ 1) over a circular cylinder (Khan, et al. [3]).

\section{Heat Transfer}

The second parameter of interest is the dimensionless average heat transfer coefficient, $N u_{D}$ for large Prandtl numbers. This parameter is determined by integrating Eq. (7) from the cylinder surface to the thermal boundary layer edge. Assuming the presence of a thin thermal boundary layer $\delta_{T}$ along the cylinder surface, the energy integral equation for the isothermal boundary condition can be written as

$$
\frac{d}{d s} \int_{0}^{\delta_{T}}\left(T-T_{a}\right) u d \eta=-\left.\alpha \frac{\partial T}{\partial \eta}\right|_{\eta=0}
$$

Using velocity and temperature profiles Eqs. (14) and (16), and assuming $\zeta=\delta_{T} / \delta<1$, Eq. (46) can be simplified to

$$
\delta_{T} \frac{d}{d s}\left[U(s) \delta_{T} \zeta(\lambda+12)\right]=90 \alpha
$$

This equation can be rewritten separately for the two regions (Fig. 1), i.e.

$$
\delta_{T} \frac{d}{d s}\left[U(s) \delta_{T} \zeta\left(\lambda_{1}+12\right)\right]=90 \alpha
$$

for the region I, and

$$
\delta_{T} \frac{d}{d s}\left[U(s) \delta_{T} \zeta\left(\lambda_{2}+12\right)\right]=90 \alpha
$$

for the region II. Integrating Eqs. (48) and (49), in the respective regions, with respect to $s$, one can obtain local thermal boundary 
layer thicknesses

$$
\left(\frac{\delta_{T}(\theta)}{D}\right) \cdot \operatorname{Re}_{D p}^{1 /(n+1)} \operatorname{Pr}_{p}^{1 / 3}=\left\{\begin{array}{l}
\sqrt[3]{45 F_{1}(n, \theta)} \text { for region I } \\
\sqrt[3]{45 F_{2}(n, \theta)} \text { for region II }
\end{array}\right.
$$

where the Prandtl number, $\operatorname{Pr}_{p}$, for a power-law fluid is defined as

$$
\operatorname{Pr}_{p}=\frac{U_{a p p} D}{\alpha} \operatorname{Re}_{D p}^{-2 /(n+1)}
$$

The functions $F_{1}(n, \theta)$ and $F_{2}(n, \theta)$ in Eq. (50) are given by

$$
\left.\begin{array}{l}
\left.F_{1}(n, \theta)=\frac{f_{1}(\theta)}{\sin ^{2} \theta\left(\lambda_{1}+12\right)^{2}}\left[\frac{2^{n-2} n \lambda_{1} \sin ^{n} \theta}{\sin 2 \theta\left(\frac{\lambda_{1}+12}{6}\right)^{1-n}}\right]^{\frac{1}{n+1}}\right]^{1 /(n+1)} \\
F_{2}(n, \theta)=\frac{f_{3}(\theta)}{\sin ^{2} \theta}\left[\frac{2^{n-2} n \lambda_{2} \sin ^{n} \theta}{\sin 2 \theta\left(\frac{\lambda_{2}+12}{6}\right)^{1-n}}\right]^{\frac{1}{6}}[
\end{array}\right\}
$$

with

$$
\left.\begin{array}{l}
f_{1}(\theta)=\int_{0}^{\theta} \sin \theta\left(\lambda_{1}+12\right) d \theta \\
f_{2}(\theta)=\int_{\theta_{1}}^{\theta_{s}} \sin \theta\left(\lambda_{2}+12\right) d \theta \\
f_{3}(\theta)=\frac{f_{1}(\theta)}{\lambda_{1}+12}+\frac{f_{2}(\theta)}{\lambda_{2}+12}
\end{array}\right\}
$$

The local heat transfer coefficients, for the isothermal boundary condition, in both the regions can be written as

$$
h_{1}(\theta)=\frac{3 k_{f}}{2 \delta_{T_{1}}} \quad \text { and } \quad h_{2}(\theta)=\frac{3 k_{f}}{2 \delta_{T_{2}}}
$$

Thus the dimensionless local heat transfer coefficients, for both the regions, can be written as

$$
\frac{\left.N u_{D}(\theta)\right|_{\text {isothermal }}}{\operatorname{Re}_{D p}^{1 /(n+1)} \operatorname{Pr}_{p}^{1 / 3}}=\frac{3}{2}\left\{\begin{array}{l}
\sqrt[3]{\frac{1}{45 F_{1}(n, \theta)}} \text { for region I } \\
\sqrt[3]{\frac{1}{45 F_{2}(n, \theta)}} \text { for region II }
\end{array}\right.
$$

The average heat transfer coefficient is defined as

$$
\begin{aligned}
h & =\frac{1}{\pi} \int_{0}^{\pi} h(\theta) d \theta \\
& =\frac{1}{\pi}\left\{\int_{0}^{\theta_{s}} h(\theta) d \theta+\int_{\theta_{s}}^{\pi} h(\theta) d \theta\right\}
\end{aligned}
$$

The integral analysis is unable to predict local heat transfer values from separation point to the rear stagnation point (second integral on R.H.S.). The experiments (Žukauskas and Žiugžda [39], Fand and Keswani [40], and Nakamura and Igarashi [41] among others for Newtonian fluids and Rao [27] for NonNewtonian fluids) show that the heat transfer from the rear portion of the cylinder increases with Reynolds numbers. For Newtonian fluids, Van der Hegge Zijnen [42] demonstrated that the average heat transferred from the rear portion of the cylinder can be determined from $N u_{D}=0.001 R e_{D}$ that shows the weak dependence of average heat transfer from the rear portion of the cylinder on Reynolds numbers. Same weak dependence can be observed from Rao [27] experiments for non-Newtonian fluids. In order to include the share of heat transfer from the rear portion of the cylinder, the local heat transfer coefficients are integrated upto the separation point and averaged over the whole surface, that is

$$
\begin{aligned}
h & =\frac{1}{\pi} \int_{0}^{\theta_{s}} h(\theta) d \theta \\
& =\frac{1}{\pi}\left\{\int_{0}^{\theta_{1}} h_{1}(\theta) d \theta+\int_{\theta_{1}}^{\theta_{s}} h_{2}(\theta) d \theta\right\}
\end{aligned}
$$

Using Eqs. (52) - (54), Eq. (57) was solved for different values of $n$ and the results were correlated in to a single correlation as a function of $n, \operatorname{Re}_{D p}$, and $\operatorname{Pr}_{p}$ :

$$
\left.N u_{D}\right|_{\text {isothermal }}=0.593 n^{-0.17} \operatorname{Re}_{D p}^{1 /(n+1)} \operatorname{Pr}_{p}^{1 / 3}
$$

For the isoflux boundary condition, the energy integral equation can be written as

$$
\frac{d}{d s} \int_{0}^{\delta_{T}}\left(T-T_{a}\right) u d \eta=\frac{q}{\rho c_{p}}
$$

Assuming constant heat flux and thermophysical properties and using Eqs. (14) and (17), Eq. (59) can be simplified to

$$
\frac{d}{d s}\left[U(s) \delta_{T}^{2} \zeta(\lambda+12)\right]=90 \frac{k_{f}}{\rho c_{p}}
$$


Rewriting Eq. (60) for the two regions in the same way as Eq. (47), one can obtain local thermal boundary layer thicknesses $\delta_{T_{1}}$ and $\delta_{T_{2}}$ under isoflux boundary condition. The local surface temperatures for the two regions can then be obtained from temperature distribution

$$
\Delta T_{1}(\theta)=\frac{2 q \delta_{T_{1}}}{3 k_{f}} \quad \text { and } \quad \Delta T_{2}(\theta)=\frac{2 q \delta_{T_{2}}}{3 k_{f}}
$$

The local heat transfer coefficient can now be obtained from its definition as

$$
h_{1}(\theta)=\frac{q}{\Delta T_{1}(\theta)} \quad \text { and } \quad h_{2}(\theta)=\frac{q}{\Delta T_{2}(\theta)}
$$

which give the local Nusselt numbers for the cross flow over a cylinder with constant flux

$$
\frac{\left.N u_{D}(\theta)\right|_{\text {isoflux }}}{\operatorname{Re}_{D p}^{1 /(n+1)} \operatorname{Pr}^{1 / 3}}=\frac{3}{2}\left\{\begin{array}{l}
\sqrt[3]{\frac{2}{45 G_{1}(n, \theta)}} \text { for region I } \\
\sqrt[3]{\frac{2}{45 G_{2}(n, \theta)}} \text { for region II }
\end{array}\right.
$$

where

$$
\left.\begin{array}{l}
G_{1}(n, \theta)=\frac{\theta}{\sin \theta\left(\lambda_{1}+12\right)}\left[\frac{2^{n-2} n \lambda_{1} \sin ^{n} \theta}{\sin 2 \theta\left(\frac{\lambda_{1}+12}{6}\right)^{1-n}}\right]^{\frac{1}{n+1}} \\
G_{2}(n, \theta)=\frac{g(\theta)}{\sin \theta}\left[\frac{2^{n-2} n \lambda_{2} \sin ^{n} \theta}{\sin 2 \theta\left(\frac{\lambda_{2}+12}{6}\right)^{1-n}}\right]^{\frac{1}{n+1}}
\end{array}\right\}
$$

with

$$
g(\theta)=\left[\frac{\theta}{\lambda_{1}+12}+\frac{\theta-\pi / 2}{\lambda_{2}+12}\right]
$$

Following the same procedure for the average heat transfer coefficient as mentioned above, one can obtain the average Nusselt number for an isoflux cylinder as

$$
\left.N u_{D}\right|_{\text {isoflux }}=0.627 n^{-0.19} \operatorname{Re}_{D p}^{1 /(n+1)} \operatorname{Pr}_{p}^{1 / 3}
$$

Combining the results for both thermal boundary conditions, we have

$$
\frac{N u_{D}}{\operatorname{Re}_{D p}^{1 /(n+1)} \operatorname{Pr}_{p}^{1 / 3}}= \begin{cases}0.593 n^{-0.17} & \text { for UWT } \\ 0.627 n^{-0.19} & \text { for UWF }\end{cases}
$$

These correlations agree with the heat transfer coefficients for a Newtonian fluid $(n=1)$ over a circular cylinder (Khan, et al. [3]).

\section{RESULTS AND DISCUSSION \\ Flow Characteristics}

The dimensionless local shear stress, $C_{f}$, is plotted in Fig. 4 for different power-law fluids. It shows that $C_{f}$ is zero at the stagnation point for each fluid and reaches a maximum at $\theta \approx$ $58^{\circ}$.

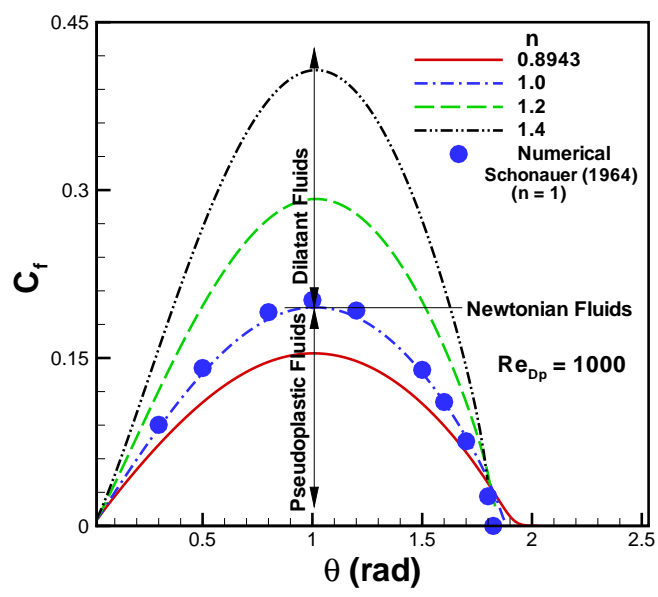

Figure 4. Effect of Power Index $n$ on Skin Friction for a Circular Cylinder.

The increase in shear stress is caused by the deformation of the velocity profiles in the boundary layer, a higher velocity gradient at the wall and a thicker boundary layer. In the region of decreasing $C_{f}$ preceeding the separation point, the pressure gradient decreases further and finally $C_{f}$ falls close to zero around the separation point, where boundary-layer separation occurs. Beyond this point, $C_{f}$ remains close to zero up to the rear stagnation point. It also shows that the skin friction increases with the increase in power-law index $n$. Thus pseudoplastic (shear-thinning) fluids offer less skin friction than dilatant (shear-thickening) fluids which is in accordance with the numerical results of Agarwal et. al [33]. The results of Newtonian fluids $(n=1)$ are compared 
with the numerical data of Schönauer [43], which shows good agreement for the entire range.

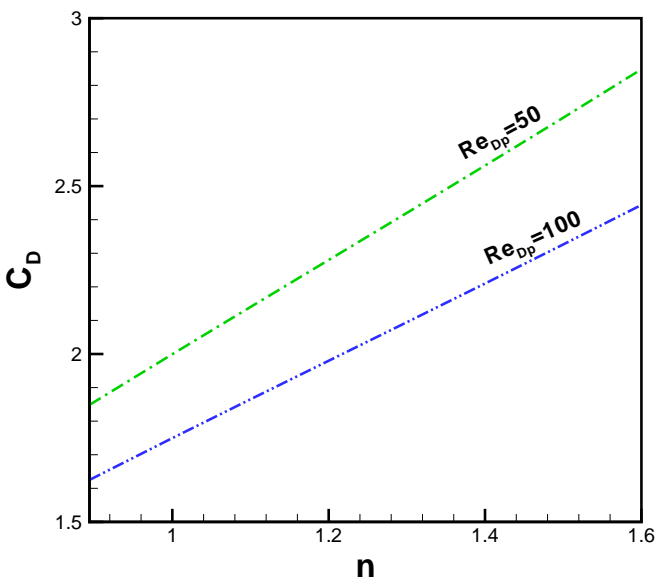

Figure 5. Effect of $n$ on Drag Coefficients for a Circular Cylinder.

The variation of the total drag coefficient $C_{D}$ with the powerlaw index $n$ for different Reynolds numbers is illustrated in Fig. 5. It shows that for a given Reynolds number, the drag coefficient $C_{D}$ increases linearly with $n$ and for a given fluid it decreases with the increase in Reynolds number. The drag coefficients for a pseudoplastic fluids are found to be lower than dilatant fluids.

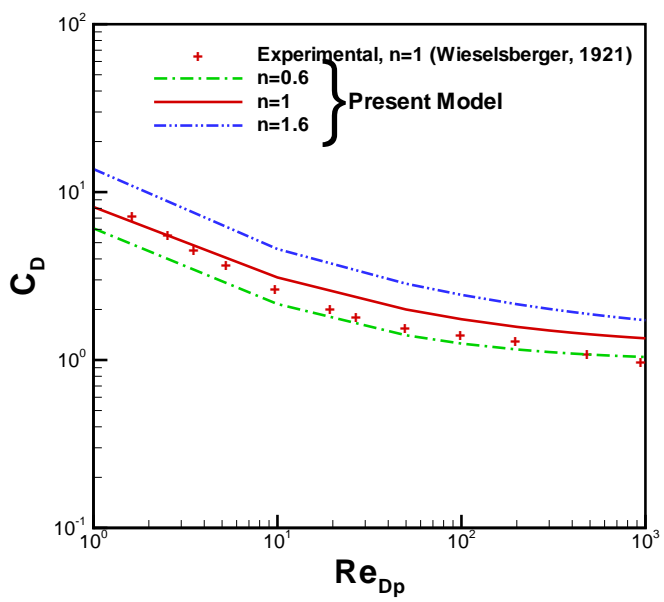

Figure 6. Effect of $R e_{D p}$ on Drag Coefficients for a Circular Cylinder.

The effect of Reynolds number on the drag coefficients for different fluids is shown in Fig. 6. Since there are no other experimental/numerical results to compare with pseudoplastic/dilatant fluids, comparisons are made with the experimental results of Wieselsberger for the air $(n=1)$ only. The comparison shows good agreement with the non-Newtonian case.

\section{Heat Transfer Characteristics}

The heat transfer parameter (HTP) $N u_{D} / \operatorname{Re}_{D p}^{1 /(n+1)} \operatorname{Pr}_{p}^{1 / 3}$ is presented in Fig. 7 for both the isothermal and isoflux boundary conditions. It shows that HTP decreases with the increase in the power law index $n$. Thus pseudoplastic fluids transfer more heat than dilatant fluids for the same thermal boundary condition. The isoflux boundary condition gives a higher heat transfer coefficient for both types of fluids.

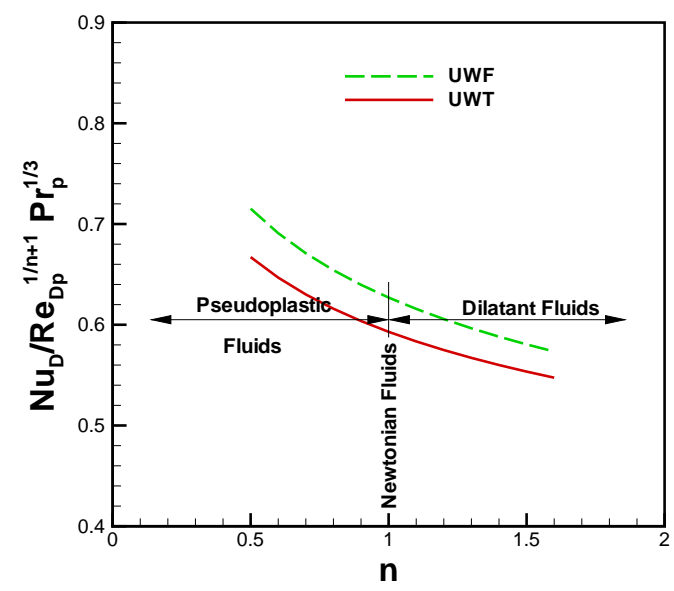

Figure 7. Comparison of Heat Transfer Parameters for Isothermal and Isoflux Thermal Boundary Conditions.

The average heat transfer coefficients $N u_{D} / \operatorname{Pr}_{p}^{1 / 3}$ versus $R e_{D p}$ are presented in Fig. 8 for different fluids. Experimental results of Takahashi et al. (1977) and Mizushina et al. (1978) for different CMC (Carboxy Methyl Cellulose) solutions are compared with the present model for isothermal boundary condition. The comparison is found to be in good agreement for all fluids.

Figure 9 shows the comparison of the average heat transfer coefficients $N u_{D} / P r_{p}^{1 / 3}$ versus $R e_{D p}$ for Newtonian fluids. Here, the experimental results of Hilpert (1933) and McAdams (1939) for air are compared with the present model for $n=1$. The results are found to be in good agreement for the entire range of Reynolds numbers. 


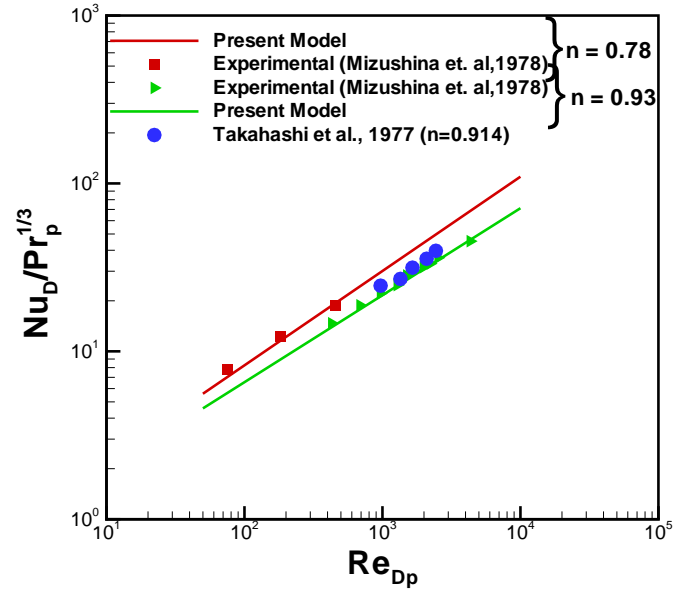

Figure 8. Comparison of Heat Transfer Coefficients from an Isothermal Circular Cylinder to CMC Solutions.

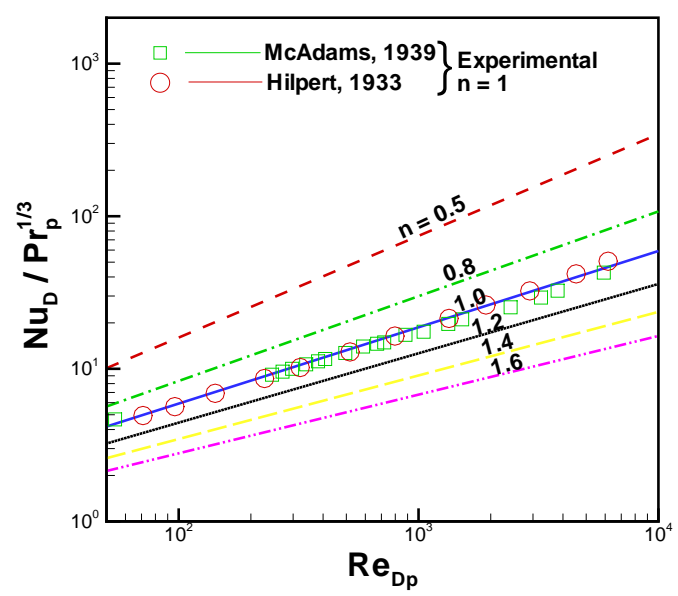

Figure 9. Comparison of Heat Transfer Coefficients for a Circular Cylinder.

\section{CONCLUSIONS}

An integral approach is employed to investigate the fluid flow and heat transfer from an isolated circular cylinder submerged in power-law fluids. Closed form solutions are developed for both the drag and heat transfer coefficients in terms of generalized Reynolds and Prandtl numbers. The correlations of heat transfer are developed for both isothermal and isoflux boundary conditions. It is found that pseudoplastic fluids offer less skin friction and higher heat transfer coefficients than dilatant fluids. Furthermore, the drag coefficients decrease and the heat transfer increases with the decrease in power-law index. It is shown that the present results are in good agreement with the experimen- tal results for the full laminar range of Reynolds numbers in the absence of free stream turbulence and blockage effects.

\section{ACKNOWLEDGMENT}

The authors gratefully acknowledge the financial support of Natural Sciences and Engineering Research Council of Canada and the Center for Microelectronics Assembly and Packaging.

\section{REFERENCES}

[1] Cho, Y. I. and Hartnett, J. P., 1985, "Non-Newtonian Fluids," Handbook of Heat Transfer Applications, Second edition, Chapter 2, McGraw-Hill Book Company, New York.

[2] Chhabra, R. P. and Richardson, J. F., 1999, "Non-Newtonian Flow in the Process Industries: Fundamentals and Engineering Applications," Butterworth-Heinemann, Great Britain.

[3] Khan, W. A., Culham, J. R., and Yovanovich, M. M., "Fluid Flow Around and Heat Transfer From an Infinite Circular Cylinder," ASME Journal of Heat Transfer (in press).

[4] Acrivos, A., Shah, M. J., and Petersen, E. E., 1960, "Momentum and Heat Transfer in Laminar Boundary-Layer Flows of Non-Newtonian Fluids Past External Surfaces," A.I.Ch.E. Journal, Vol. 6, No. 2, pp.312-317.

[5] Schowalter, W. R., 1960, "Application of Boundary-Layer Theory to Power-Law Pseudo-Plastic Fluids: Similar Solutions," A.I.Ch.E. Journal, Vol. 6, No. 1, pp.24-28.

[6] Shah, M. J., 1962, Petersen, E. E., and Acrivos, A., "Heat Transfer from a Cylinder to a Power-Law Non-Newtonian Fluid,” A. I. Ch.E. Journal, Vol. 8, No. 4, pp.542-549.

[7] Acrivos, A., Shah, M. J., and Petersen, E. E., 1965, "On Solution of Two-Dimensional Boundary-Layer Flow Equations for Non-Newtonian Power-Law Fluid," Chemical Engineering Science, Vol. 20, No. 2, pp. 101-105.

[8] Lee, S. Y., and Ames, W. F., 1966, "Similarity Solutions for Non-Newtonian Fluids," A.I.Ch.E. Journal, Vol. 12, No. 4, pp. 700-708.

[9] Bizzle, G. D. and Slattery, J. C., 1962, "Non-Newtonian Boundary-Layer Flow," Chemical Engineering Science, Vol. 17, pp.777-782.

[10] Wolf, C. J. and Szewczyk, A. A., 1966, "Laminar Heat Transfer to Non-Newtonian Fluids from Arbitrary Cylinders," Proceedings of the Third IHTC, Chicago, Illinois, August 7-12, Volume 1.

[11] Serth, R. W., and Kiser, K. M., 1967, "A Solution of the Two-Dimensional Boundary-Layer Equations for an Ostwald-de Waele Fluid," Chemical Engineering Science, Vol. 22, pp. 945-956.

[12] Lin, F. N. and Chern, S. Y., 1979, "Laminar Boundary ayer Flow of Non-Newtonian Fluid," International Journal of Heat and Mass Transfer, Vol. 22, pp. 1323-1329. 
[13] Kim, H. W., Jeng, D. R., and DeWitt, K. J., 1983, “Momentum and Heat Transfer in Power-Law Fluid Flow Over Two-Dimensional or Axisymmetrical Bodies," International Journal of Heat and Mass Transfer, Vol. 26, No. 2, pp. 245259.

[14] Mizushina, T. and Usui, H., 1978, "Approximate Solution of the Boundary Layer Equations for the Flow of a Non-Newtonian Fluid Around a Cylinder," Heat Transfer: Japanese Research, Vol. 7, No. 2, pp. 83-92.

[15] Nakayama, A., Shenoy, A. V., and Koyama, H., 1986, “An Analysis for Forced Convection Heat Transfer from External Surfaces to Non-Newtonian Fluids," Warme-und Stoffubertragung, Vol. 20, pp. 219-227.

[16] Shenoy, A. V., and Nakayam, A., 1986, "Forced Convection Heat Transfer fro Axisymmetric Bodies to NonNewtonian Fluids," Canadian Journal of Chemical Engineering, Vol. 64, pp. 680-686.

[17] Nakayama, A., 1986, "Integral Methods for Forced Convection Heat Transfer in Power-Law Non-Newtonian Fluids," Encyclopedia of Fluid Mechanics: Rheology and NonNewtonian Flows, Vol. 7, pp. 305-339, Houston, USA:Gulf.

[18] Anderson, H. I., 1988, "The Nakayama-Koyama Approach to Laminar Forced Convection Heat Transfer to Power-Law Fluids," International Journal of Heat and Fluid Flow, Vol. 9, No. 3, pp. 343-346.

[19] Luikov, A. V., Schulman, Z. P., and Berkovsky, B. M., 1966, "Heat and Mass Transfer in a Boundary Layer of Non-Newtonian Fluids," Proceedings of the Third IHTC, Chicago, Illinois, August 7-12, Volume 1.

[20] Luikov, A. V., Schulman, Z. P., and Puris, B. I., 1969a, "Mass Transfer of Cylinder in Forced Flow of NonNewtonian Viscoelastic Fluid," Heat Transfer-Soviet Research, Vol. 1, No. 1, pp. 121-32.

[21] Luikov, A. V., Schulman, Z. P., and Puris, B. I., 1969b, "External Convective Mass Transfer in Non-Newtonian Fluid,' International Journal of Heat and Mass Transfer, Vol. 12, pp. 377-391.

[22] James, D. F., and Acosta, A. J., 1970, "The Laminar Flow of Dilute Polymer Solutions Around Circular Cylinders," Journal of Fluid Mechanics, Vol. 42, Part 2, pp. 269-288.

[23] Takashi, K., Maeda, M., and Ikai, S., 1977, "Experimental Study of Heat Transfer from a Cylinder Submerged in a Non-Newtonian Fluid," die dem Nahenungsansatz von K. Pohlhausen genugen, Lilenthal Bericht 510, p. 335-339.

[24] Mizushina, T., Usui, H., Veno, K., and Kato, T., 1978, "Experiments of Pseudoplastic Fluid Cross Flow Around a Circular Cylinder,' Heat Transfer: Japanese Research, Vol. 7, No. 3, pp. 92-101.

[25] Kumar, S., Mall, B. K., and Upadhyay, S. N., 1980, “On the Mass Transfer in Non-Newtonian Fluids: II Transfer fro Cylinders to Power-Law Fluids," Letters in Heat and Mass Transfer, Vol. 7, pp. 55-64.
[26] Ghosh, U. K., Gupta, S. N., Kumar, S., and Upadhay, S. N., 1986, "Mass Transfer in Cross Flow of Non-Newtonian Fluid Around a Circular Cylinder," International Journal of Heat and Mass Transfer, Vol. 29, No. 6, pp. 955-960.

[27] Rao, B. K., 2000, "Heat Transfer to Non-Newtonian Flows Over a Cylinder in Cross Flow," International Journal of Heat and Fluid Flow, Vol. 21, pp. 693-700.

[28] Coelho, P. M., and Pinho, F. T., 2003, "Vortex Shedding in Cylinder Flow of Shear-Thinning Fluids: I. Identification and Demarcation of Flow Regimes," Journal of NonNewtonian Fluid Mechanics, Vol. 110, pp. 143-176.

[29] Coelho, P. M., and Pinho, F. T., 2003, "Vortex Shedding in Cylinder Flow of Shear-Thinning Fluids: II. Flow Characteristics," Journal of Non-Newtonian Fluid Mechanics, Vol. 110, pp. 177-193.

[30] Coelho, P. M., and Pinho, F. T., 2004, "Vortex Shedding in Cylinder Flow of Shear-Thinning Fluids: III. Pressure Characteristics," Journal of Non-Newtonian Fluid Mechanics, Vol. 121, pp. 55-68.

[31] D’Alessio, S. J. D., and Pascal, J. P., 1996, "Steady Flow of a Power-Law Fluid Past a Cylinder," Acta Mechanica, Vol. 117, pp. 87-100.

[32] Chhabra, R. P., Soares, A. A., and Ferreira, J. M., 2004, "Steady Non-Newtonian Flow Past a Circular Cylinder: A Numerical Study," Acta Mechanica, Vol. 172, pp. 1-16.

[33] Agarwal, M., Chhabra, R. P., and Eswaran, V., 2002, "Laminar Momentum and Thermal Boundary Layers of Power-Law Fluids Over a Slender Cylinder," Chemical Engineering Science, Vol. 57, pp.1331-1341.

[34] Khan, W. A., 2004, "Modeling of Fluid Flow and Heat Transfer for Optimization of Pin-Fin Heat Sinks," Ph. D. Thesis, Department of Mechanical Engineering, University of Waterloo, Canada.

[35] Pohlhausen, K., 1921, "Zur Näherungsweise Integration der Differential Gleichung der Laminaren Reibungschicht," Zeitschrift für angewandte Mathematic und Mechanic, Vol. 1, pp. 252-268.

[36] Schlichting, H., 1979, Boundary Layer Theory, 7th Edition., McGraw-Hill, New York.

[37] Walz, A., 1941, "Ein neuer Ansatz für das Greschwindligkeitsprofil der laminaren Reibungsschicht," LilienthalBericht 141, p. 8.

[38] Shibu, S., Chhabra, R. P., and Eswaran, V., 2001, "PowerLaw Fluid Flow Over a Bundle of Cylinders at Intermediate Reynolds Numbers," Chemical Engineering Science, Vol. 56, pp. 5545-5554.

[39] Žukauskas, A. and Žiugžda, J., 1985, Heat Transfer of a Cylinder in Crossflow, Hemisphere Publishing Corporation, New York.

[40] Fand, R. M., and Keswani, K. K., 1972, “A Continuous Correlation Equation for Heat Transfer From Cylinders to Air in Crossflow for Reynolds Numbers From $10^{-2}$ to $2 \times$ 
$10^{5}$, International Journal of Heat and Mass Transfer, Vol. 15 , pp. 559-562.

[41] Nakamura, H., and Igarashi, T., 2004, "Variation of Nusselt Number with Flow Regimes Behind a Circular Cylinder for Reynolds Numbers from 70 30000," International Journal of Heat and Mass Transfer, Vol. 47, pp. 5169-5173.

[42] Van der Hegge Zijnen, B. G., 1956, "Modified Correlation Formulae for Heat Transfer by Natural and Forced Convection from Horizontal Cylinders," Applied Scientific Research-A, Vol. 6, No. 2-3, pp. 129-140.

[43] Schönauer, W., 1964, "Ein Differenzenverfahren zur Lösung der Grenzschichtgleichung für stationäre, laminare, inkompressible Strömung,” Ing.-Arch., Vol. 33, p. 173. 